\title{
Objetos de Aprendizagem e contribuições para Educação Matemática: mapeamento teórico de artigos acadêmicos
}

\section{Learning Objects and Mathematical Education contributions: mapping of academic articles}

\author{
Bruno Resende \\ Pontifícia Universidade Católica do Rio Grande do Sul (PUCRS) \\ Programa de Pós-Graduação em Educação em Ciências e Matemática \\ Porto Alegre, RS, Brasil \\ bruno.resende@acad.pucrs.br
}

Isabel Cristina Machado de Lara

Pontifícia Universidade Católica do Rio Grande do Sul (PUCRS)

Programa de Pós-Graduação em Educação em Ciências e Matemática

Porto Alegre, RS, Brasil

isabel.lara@pucrs.br

\section{Informações do Artigo}

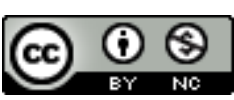

Histórico do Artigo

Submissão: 29 de agosto de 2018.

Aceite: 25 de dezembro de 2018.

\section{Palavras-chave}

Objetos de Aprendizagem

Ensino Matemática

Ensino e Aprendizagem

\begin{abstract}
Resumo
O presente artigo objetiva apresentar um mapeamento teórico sobre o modo como os Objetos de Aprendizagem (OA) vêm sendo utilizados para o ensino e a aprendizagem e suas contribuições no campo da Educação Matemática. Buscou-se produções de artigos entre os anos de 2010 e 2017 por meio do Banco de Periódicos da Coordenação de Aperfeiçoamento de Pessoal de Nível Superior (CAPES). Durante o procedimento de identificação das produções, foram obtidos 23 artigos sendo oito escolhidos para leitura e análise. Mediante a leitura dos trabalhos, identificou-se o tema, objetivos, metodologia, referenciais teóricos, público-alvo assim como as principais considerações. Os OA foram evidenciados como ferramenta mediadora de conhecimento, inovadora de conteúdos, além de possuir ampla relevância pedagógica que pode contribuir positivamente na interação com o professor apesar de existir a necessidade da aproximação dos docentes com esses recursos.
\end{abstract}

\section{Abstract}

The present article aims to present a theoretical mapping about the way learning objects $(O A)$ are being used for teaching and learning and their contributions in the field of Mathematics Education. Productions of articles were sought between 2010 and 2017 through the database of journal of the Coordenação de Aperfeiçoamento de Pessoal de Nível Superior (CAPES). During the production identification procedure, 23 articles were obtained, eight of which were chosen for reading and analysis. Through the reading of the works, we identified the theme, objectives, methodology, theoretical references, target audience as well as the main considerations. The Learning Objects were evidenced as a mediator of knowledge, innovator of contents, besides having broad pedagogical relevance that can contribute positively in the interaction with the teacher although there is a need to bring teachers closer to these resources.

\section{Introdução}

As tecnologias na educação têm crescido em discursos e estudos no âmbito da Educação em Ciências e Matemática. Desde, aproximadamente, a década de 2010, está ocorrendo um crescimento de produções acerca dos Objetos de Aprendizagem (OA) como um novo modo de contribuição para aprendizagens diversas. 
Diante disso, realiza-se uma investigação sobre os OA utilizados para o ensino e aprendizagem na área da Educação Matemática. Assim, esta pesquisa contempla as possibilidades desses recursos digitais das Tecnologias de Informação e Comunicação (TIC) no processo de transmissão e construção do conhecimento.

O objetivo do trabalho é apresentar um mapeamento teórico em relação às mais recentes contribuições acadêmicas contidas em artigos de periódicos sobre OA no contexto da Educação Matemática.

Para analisar essas produções, foram consideradas as concepções sobre o uso de Objetos de Aprendizagem nos últimos anos em relação as suas contribuições para o ensino, aprendizagem, formação de professores e práticas educacionais.

Portanto, para levantar as perspectivas, significados, definições, problemas, entre outras características do tema abordado, adota-se a metodologia do mapeamento teórico (BIEMBENGUT, 2008) na busca dos artigos acadêmicos mais relevantes e mais recentes.

Para identificar os resultados obtidos na procura por artigos produzidos dentre os anos de 2010 e 2017, o ambiente virtual de periódicos da Coordenação de Aperfeiçoamento de Pessoal de Nível Superior (CAPES) foi escolhido. Assim, para compor o mapa teórico, a procura foi composta pelo vocábulo preciso "Objetos de Aprendizagem" com a adição das palavras Educação, Matemática e Tecnologia. A partir dessa busca, diversos artigos emergiram, sendo selecionados apenas oito para leitura na íntegra, os quais mais se aproximavam ao objetivo deste estudo.

\section{Conceitos e definições}

Conforme Biembengut (2008), temos que esclarecer os conceitos e as definições sobre os termos presentes no mapeamento. Ainda segundo o autor, a

[...] compreensão dos conceitos e das definições nos auxiliará não apenas a identificar quais deles foram utilizados em cada uma das pesquisas em que mapearemos na sequência, como também elaborarum outro conceito e uma outra definição, ou, então, a adotar algum deles para pesquisa que pretendemos conduzir (BIEMBENGUT, 2008, p. 90).

Em relação ao tema deste trabalho, serão definidos, portanto, Objetos de Aprendizagem, Tecnologias de Informação e Comunicação e Educação Matemática, buscando as concepções e significados dos vocábulos na perspectiva de compreender conceitos específicos contidos nos artigos pesquisados para uma posterior análise desses objetos de estudo.

\subsection{Tecnologias de Informação e Comunicação na Educação Matemática}

As TIC são “[...] recursos tecnológicos que proporcionam por meio das funções de software e telecomunicações, a automação e comunicação dos processos de negócios, da pesquisa científica e de ensino e aprendizagem." (OLIVEIRA, 2015, p. 78). 
Com os avanços tecnológicos, a sociedade apresenta uma mudança considerável em seu âmbito sociocultural. Diante disso, as TIC provocam mudanças de ações e hábitos no modo como as pessoas encaram, veem, vivem e aprendem o mundo (PAULIN, 2015).

Assim, a Educação Matemática não fica imune a essas alterações presentes na sociedade. As TIC trouxeram novos modos de ensino e de aprendizagem, possibilitando novos recursos no cenário educacional.

A Educação Matemática se constitui como "[...] uma região de inquérito em torno de questionamentos específicos e busca procedimentos apropriados às ações de educar e ensinar Matemática." (BICUDO et al., 2005, p. 13). Desse modo, a Educação Matemática é uma área que se dedica ao estudo dos processos de ensino e de aprendizagem de Matemática. Um dos temas de investigação desse campo é o potencial do uso da tecnologia, mais especificamente, das TIC, nas aulas de Matemática.

O computador se tornou um dos instrumentos tecnológicos mais importantes como recurso pedagógico na educação. Os softwares utilizados em sala de aula, por exemplo, interagem com o usuário por meio de uma tela com realidade bi e tridimensional. O computador trouxe, então, sofisticação na interatividade entre o ser humano e a máquina, exigindo que as pessoas se ajustassem às interfaces e à tecnologia (KIRNER; SISCOUTTO, 2007). É sinal de que há uma particularidade na sociedade contemporânea que possui a presença das TIC em quase todas as ações do homem, fazendo, assim, transformações que também influenciam na atmosfera educacional segundo os estudos de Piccoli (2006). Além disso, o autor reforça que não podemos impedir a modificação do ensino e da aprendizagem sendo "[...] preciso encaminhar discussões de operacionalização dessas mudanças." (PICCOLI, 2006, p. 22).

"Com a conexão à Internet, começaram a surgir diferentes contextos de informação, comunicação e formas de trabalho." (BAIRRAL, 2015, p. 97). Assim, a Internet proporcionou diferentes formas de Educação a Distância (EAD), como plataformas de Ambientes Virtuais de Aprendizagem (AVA).

Entre esses recursos e ferramentas que oferecem novas possibilidades para o ensino e aprendizagem, destaca-se os Objetos de Aprendizagem.

\subsection{Objetos de Aprendizagem}

Os OA têm características e conceitos vastos em relação a sua utilização para a aprendizagem. Apoiados em TIC, os OA são recursos digitais com finalidades pedagógicas (SINGO, 2014).

Segundo Fabre et al. (2003, p. 2), os "[...] objetos educacionais de aprendizagem podem ser definidos como qualquer recurso, suplementar ao processo de aprendizagem, que pode ser reusado para apoiar a aprendizagem.”. Dado que o objetivo surgiu “[...] para localizar conteúdos 
educacionais na Web para serem utilizados em diferentes cursos, ambientes virtuais de aprendizagem e plataformas [...]" (SINGO, 2014, p. 19).

Conforme Boucinha et al. (2014, p. 165), os objetos de aprendizagem proporcionam novas possibilidades no meio educacional, visto que "[...] é concebido para proporcionar ao sujeito informação, conhecimento e aprendizagem". Além disso, a qualidade na aprendizagem não está relacionada aos tipos de recursos que são utilizados, mas com a maneira como são adequadamente usados. Nessa perspectiva, os Objetos de Aprendizagem podem desenvolver e engrandecer os processos de ensino e aprendizagem pois, quando utilizados de maneira correta, são possivelmente mais eficientes que outras alternativas (FILHO; LIMA, 2014).

De acordo com Singo (2014), uma das características dos Objetos de Aprendizagem é a reusabilidade. Assim, os objetos podem ser, sem dificuldade, adaptados e flexíveis. Acrescenta, ainda, que um objeto de aprendizagem pode ser usado em âmbitos e locais diferentes.

Além da reusabilidade, conforme Fabre et al. (2003), existem outros benefícios em relação aos objetos educacionais de aprendizagem:

- Acessibilidade: possibilidade de conectar recursos educacionais em um local remoto e utilizá-los em diferentes locais;

- Interoperabilidade: possibilidade de usar elementos desenvolvidos em um local, com alguma espécie de recurso ou plataforma, em outros ambientes com outros recursos e plataformas;

- Durabilidade: possibilidade de utilização contínua quando existe avanço tecnológico.

Portanto, na perspectiva dos objetos educacionais de aprendizagem, é preciso entender que, para desenvolver um recurso tecnológico ou simplesmente escolher uma ferramenta com essas propriedades, é necessário elaboração, planejamento e esforço (TAROUCO et al., 2004). Por conseguinte, os Objetos de Aprendizagem podem trazer ótimos resultados nos processos de ensino e de aprendizagem, desde que se tenha conhecimento da utilização e da aplicação adequada dos recursos disponíveis.

$\mathrm{Na}$ seção seguinte, apresenta-se o desenvolvimento da pesquisa mediante os artigos selecionados para compor o mapeamento teórico.

\section{Mapas de pesquisas acadêmicas}

Por meio do mapeamento, é possível, segundo Biembengut (2008), realizar um exercício com o intuito de identificar, classificar e organizar as pesquisas sobre os temas que serão estudados. Para a autora, esse processo contribuiu para a obtenção de informações atuais que permitam comparação entre pesquisas existentes.

Nos próximos parágrafos, descreve-se a identificação, classificação e organização dos trabalhos escolhidos sobre o modo como os Objetos de Aprendizagem vêm sendo usados no campo da Educação Matemática. 


\subsection{Identificação}

Inicialmente, buscou-se realizar uma pesquisa dos artigos acadêmicos no período entre 2010 e 2017 com abordagem em Objetos de Aprendizagem, tecnologia e Educação Matemática. Dessa forma, foi utilizado o portal de periódicos da CAPES para identificar trabalhos publicados com os verbetes como Objetos de Aprendizagem, Educação, Matemática e Tecnologia.

O portal de periódicos da CAPES é um acervo virtual de produções científicas nacionais e internacionais que tem como um dos principais objetivos fomentar o fortalecimento da pesquisa acadêmica no Brasil por meio do acesso a conteúdos científicos online.

A biblioteca virtual permite pesquisas sobre trabalhos por um mecanismo de buscas avançadas por assunto, periódico, livro ou base de dados. Nesse mapeamento, procurou-se buscar artigos por assunto. Primeiramente, os termos pesquisados foram Objetos de Aprendizagem, Educação e Matemática. Desse modo, o resultado obtido foi de 215 artigos entre 2010 e 2017. O Mapa 1 e o Gráfico 1 ilustram o resultado.

Mapa 1 - Pesquisa inicial.

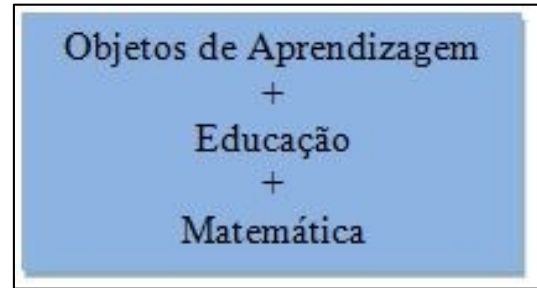

Fonte: Elaboração dos autores.

Gráfico 1 - Gráfico do primeiro resultado.

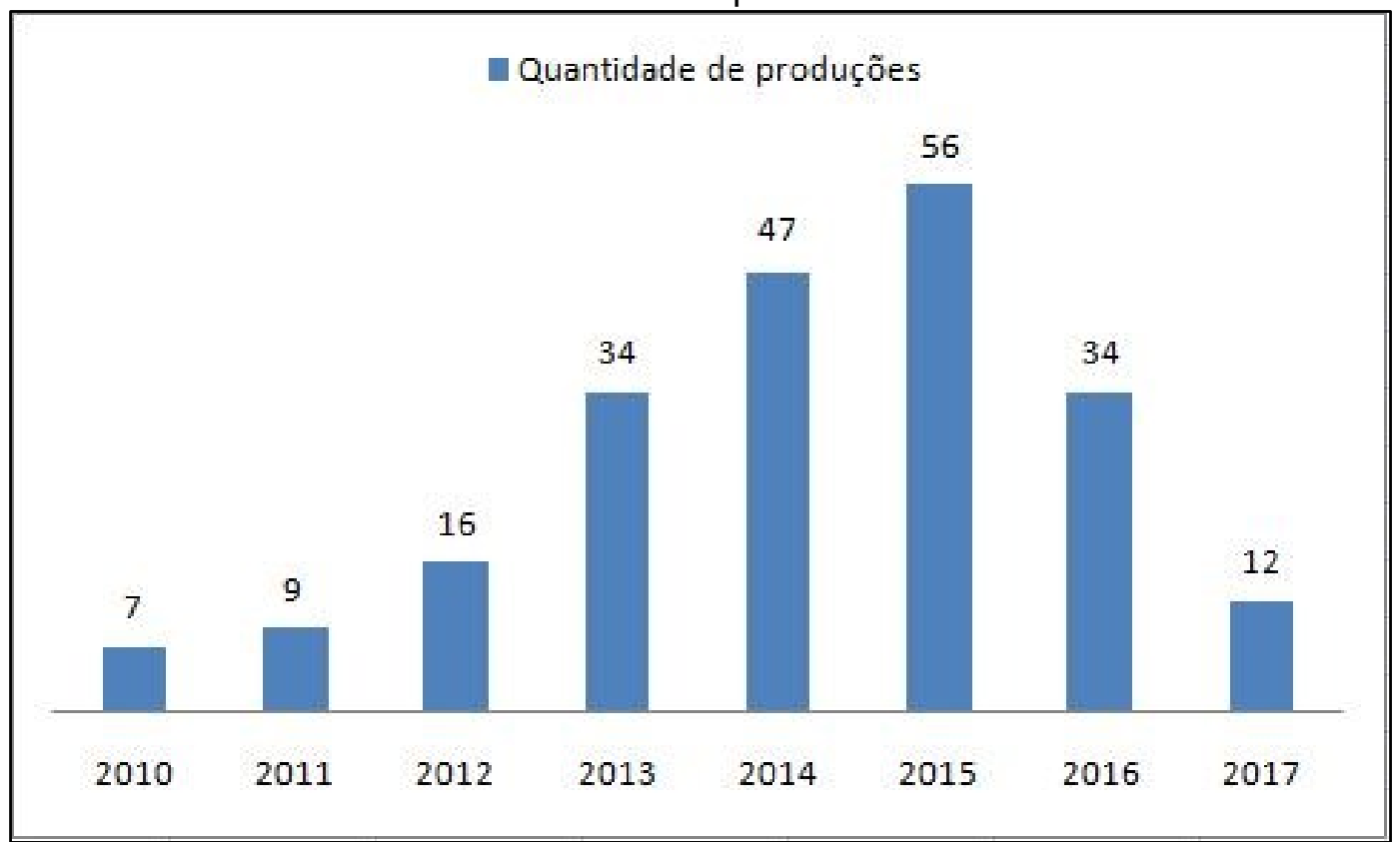

Fonte: Elaboração dos autores com base nos dados fornecidos pelo Portal de Periódicos da CAPES.

Em uma tentativa de refinamento da pesquisa, o termo Objetos de Aprendizagem foi digitado entre aspas ("'"), juntamente com Educação e Matemática. Dessa vez, o resultado retornado foi de 33 artigos no período de 2010 a 2016. Esse resultado mais enxuto aconteceu 
devido ao termo Objetos de Aprendizagem estar totalmente entre aspas, que o recurso interpreta como uma busca pelo termo completo. No Mapa 2 e no Gráfico 2 tem-se os termos e os totais das produções por ano.

Mapa 2 - Termos utilizados no refinamento da pesquisa.

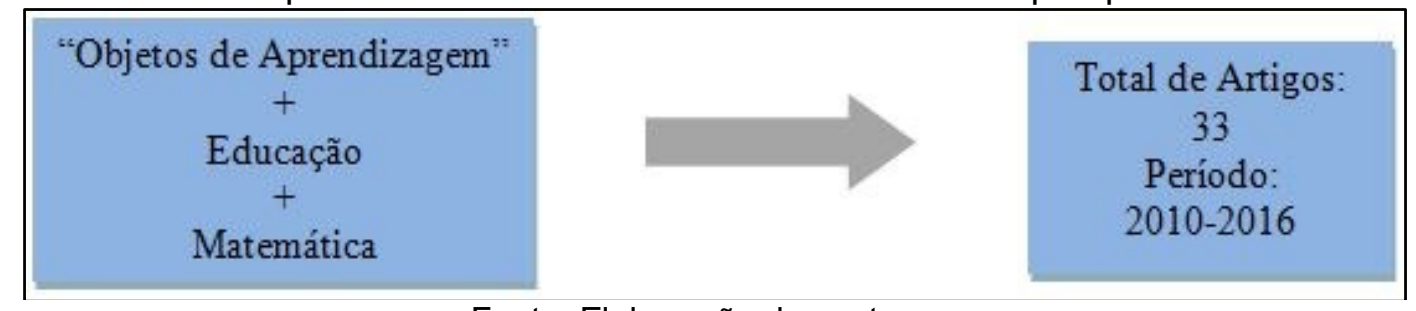

Fonte: Elaboração dos autores.

Gráfico 2 - Gráfico do refinamento da pesquisa.

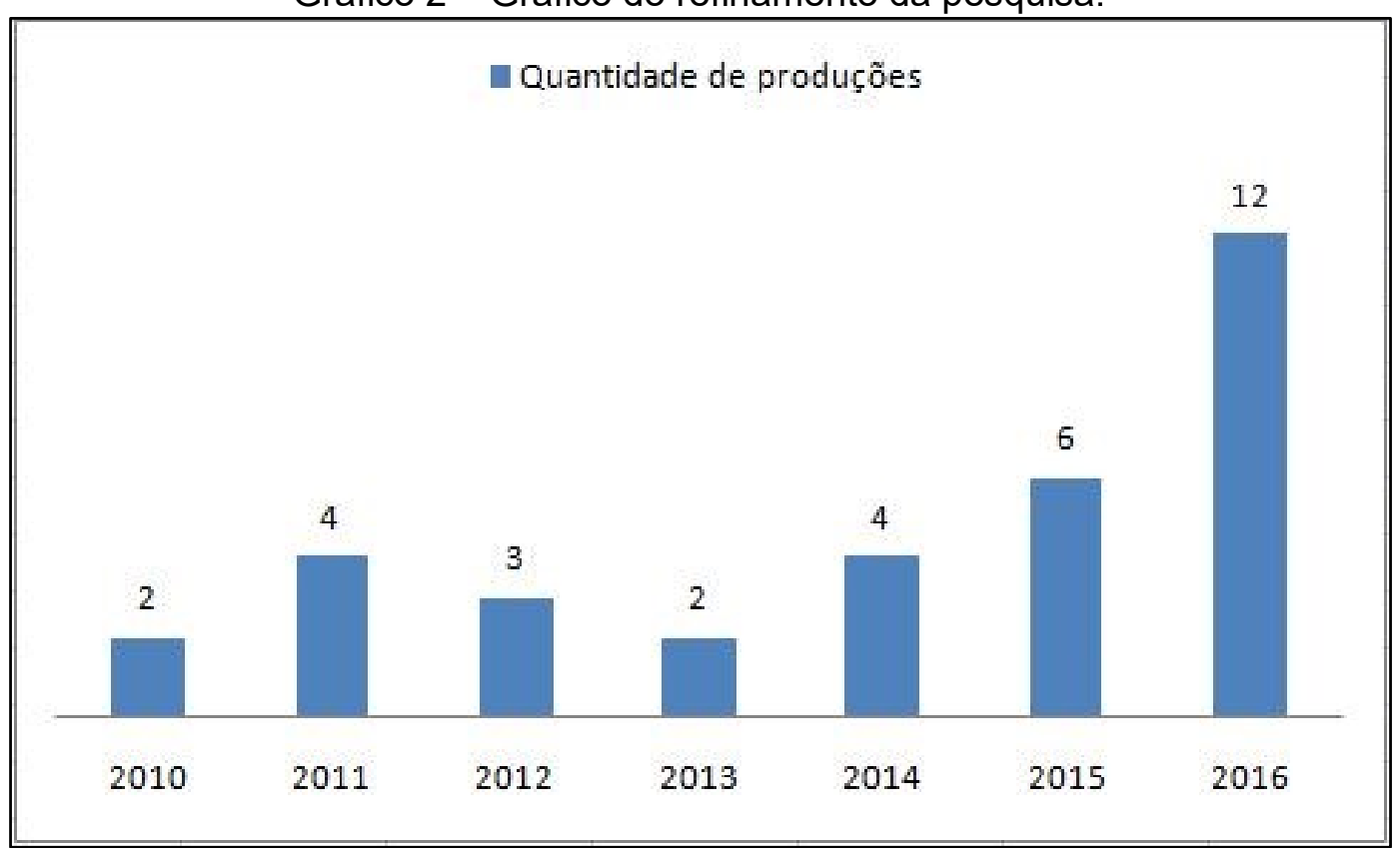

Fonte: Elaboração dos autores com base nos dados fornecidos pelo Portal de Periódicos da CAPES.

Com o intuito de aperfeiçoar a busca de trabalhos com os termos específicos, acrescentouse mais uma palavra no campo de pesquisa do Portal de Periódicos da CAPES: Tecnologia. Assim, a composição final da pesquisa por trabalhos acadêmicos ficou com os vocábulos "Objetos de Aprendizagem", Educação, Matemática e Tecnologia. Essa última procura resultou em 23 artigos entre os anos de 2010 e 2016, conforme o Mapa 3 e o Gráfico 3 ilustram.

Mapa 3 - Termos da composição final da pesquisa.

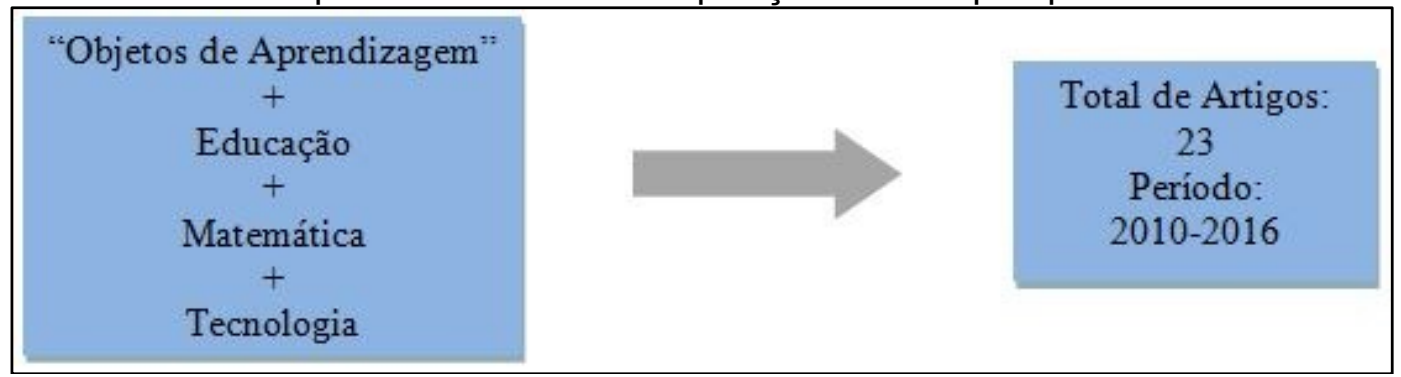

Fonte. Elaboração dos autores. 
Gráfico 3 - Gráfico da composição final da pesquisa.

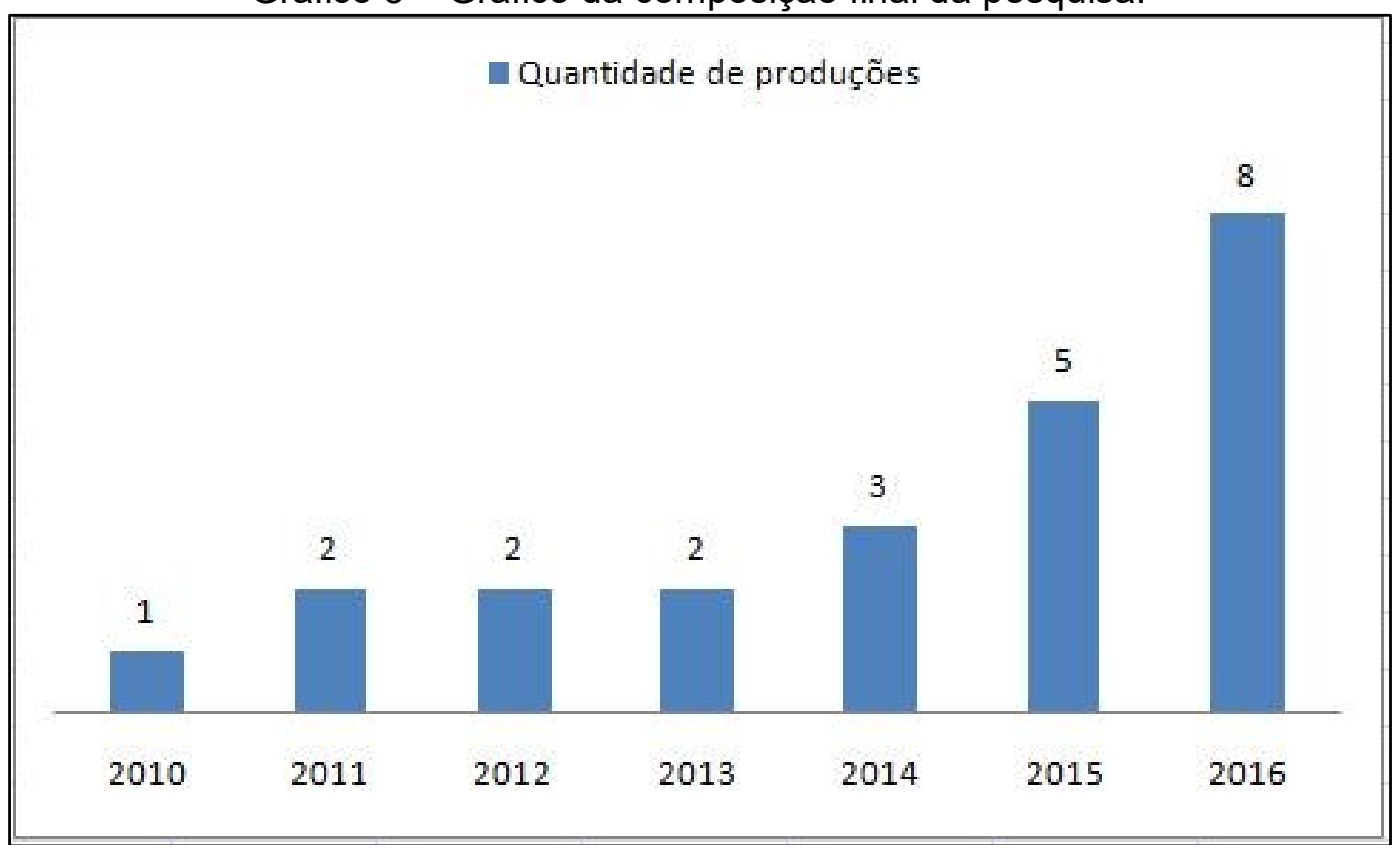

Fonte: Elaboração dos autores com base nos dados fornecidos pelo Portal de Periódicos da CAPES.

Posteriormente, no processo de análise dos artigos retornados pelo acervo digital, foram identificados trabalhos acadêmicos que não iam ao encontro do objetivo de trabalho no âmbito da Matemática e da tecnologia. Por conseguinte, foram escolhidas as produções de acordo com os contextos: Educação em Ciência e Matemática; Objetos de Aprendizagem; Tecnologia de Informação e Comunicação. Os demais trabalhos acadêmicos não foram analisados.

Portanto, oito artigos, dentre os resultados encontrados, foram escolhidos para compor o mapeamento teórico e o processo de análise. Assim, na próxima seção, destaca-se a organização das produções estudadas e analisadas.

\subsection{Classificação e organização}

No intuito de organizar e classificar os trabalhos acadêmicos escolhidos, foi elaborado o Quadro 1 com as respectivas informações: ano; título; autores; periódico. Cada artigo foi identificado com um código para facilitar sua identificação. 
Quadro 1-Quadro das produções escolhidas para compor a análise.

\begin{tabular}{|c|c|c|c|c|}
\hline Código & Autores & Título & Ano & Periódico \\
\hline A1 & $\begin{array}{l}\text { Romildo L. Sampaio; } \\
\text { Ana Rita S. Almeida. }\end{array}$ & $\begin{array}{l}\text { Aprendendo Matemática com } \\
\text { Objetos de Aprendizagem }\end{array}$ & 2010 & Ciências e Cognição \\
\hline A2 & $\begin{array}{c}\text { Maria Lúcia; } \\
\text { Moreira Gomes; } \\
\text { Nelma V. P. Barreto. }\end{array}$ & $\begin{array}{c}\text { Educaçãa a distância como } \\
\text { ferramenta de dependência e } \\
\text { reforço no ensino superior do } \\
\text { CEFET Campos: uma experiência } \\
\text { em pauta }\end{array}$ & 2011 & Vértices \\
\hline A3 & Adelaide Carreira & $\begin{array}{c}\text { Uma experiência extracurricular } \\
\text { de matemática com crianças } \\
\text { carenciadas }\end{array}$ & 2012 & $\begin{array}{l}\text { Revista Eletrônica } \\
\text { de Educação }\end{array}$ \\
\hline A4 & $\begin{array}{l}\text { E. J. Ramos; } \\
\text { L. H. Amaral. }\end{array}$ & $\begin{array}{c}\text { Relações e interações dos } \\
\text { professores de ciência e } \\
\text { matemática com as tecnologias }\end{array}$ & 2012 & Holos \\
\hline A5 & $\begin{array}{l}\text { Larissa WeyhMonzon; } \\
\text { Maria Alice Gravina. }\end{array}$ & $\begin{array}{l}\text { Uma introdução às funções de } \\
\text { variável complexa no Ensino } \\
\text { Médio: uma possibilidade através } \\
\text { do uso de animações interativas }\end{array}$ & 2013 & Bolema \\
\hline A6 & $\begin{array}{l}\text { D. L. Maia; } \\
\text { R. L. Carvalho; } \\
\text { J. A. Castro Filho; } \\
\text { E. S. Junqueira. }\end{array}$ & $\begin{array}{l}\text { Formação de professores que } \\
\text { ensinam matemática no contexto } \\
\text { da cibercultura: estudo em uma } \\
\text { escola UCA }\end{array}$ & 2014 & Holos \\
\hline A7 & $\begin{array}{l}\text { Maria Ivete Basniak; } \\
\text { Dirceu Scaldelai; } \\
\text { Celine Maria Paulek; } \\
\text { Natali Angela Felipe. }\end{array}$ & $\begin{array}{c}\text { Tecnologias digitais no ensino: } \\
\text { discussões a partir de propostas } \\
\text { desenvolvidas por licenciandos } \\
\text { envolvendo polinômios }\end{array}$ & 2015 & $\begin{array}{l}\text { Educação } \\
\text { Matemática } \\
\text { Pesquisa }\end{array}$ \\
\hline A8 & D. M. Ozelame & $\begin{array}{c}\text { Concepções de professores sobre } \\
\text { o uso de tecnologias digitais nas } \\
\text { escolas do ensino fundamental do } \\
\text { paraná: o caso do ensino das } \\
\text { ciências da natureza }\end{array}$ & 2016 & Holos \\
\hline
\end{tabular}

Fonte: Elaboração dos autores com base nos dados fornecidos pelo Portal de Periódicos da CAPES.

A partir da classificação e da organização, apresenta-se uma síntese com as ideias fundamentais das produções pesquisadas.

\section{Reconhecimento e análise}

Para compor o processo de reconhecimento e análise dos artigos selecionados, o mapeamento teórico seguiu a perspectiva de "[...] combinar vários dados ou resultados específicos em um mais geral, realizando combinações por meio de associações em função de similaridades, contrastes ou proximidade, vizinhança [...]" (BIEMBENGUT, 2008, p. 95). Primeiramente, foi feita uma síntese agrupando os objetivos, metodologia, instrumentos de coleta e público-alvo. Em seguida, o referencial teórico e as considerações dos artigos foram reunidos em categorias emergentes durante a análise.

Segundo os objetivos, verifica-se que as produções acadêmicas têm como intuito investigar a validade do uso de Objetos de Aprendizagem como recurso pedagógico mediador na cooperação entre crianças, estudar as concepções de professores sobre a utilização de tecnologias educacionais no ensino de ciências, discutir diferentes abordagens de conteúdos 
matemáticos com tecnologias digitais, abordar a ideia de um projeto de Educação a Distância com o uso de um ambiente virtual, transmitir às crianças conceitos e métodos matemáticos por meio da tecnologia como diversão, explorar como ocorrem as relações e interações dos professores de Matemática com as tecnologias, mostrar o quanto as manipulações dinâmicas dos sistemas de representação (Objetos de Aprendizagem) podem ajudar no processo de aprendizagem e analisar de que forma as interações mediadas pelas tecnologias digitais contribuem para 0 desenvolvimento profissional do professor de Matemática.

As abordagens metodológicas encontradas nos trabalhos foram: procedimentos qualitativos - quatro artigos; colaborativa - um artigo; pesquisa qualitativa e quantitativa - um artigo. No entanto, dois artigos não descreveram qual metodologia foi utilizada.

No que se refere aos principais instrumentos de coleta de informações utilizados, percebese o uso de questionário, a análise de dados armazenados em um software de computador, planos de aula, anotações feitas durante a utilização do Objeto de Aprendizagem, o uso de correio eletrônico, formulário online e fóruns de discussão.

Percebe-se que todas as atividades com tecnologias digitais aplicadas englobaram os seguintes públicos: crianças da pré-escola e quatro anos iniciais; alunos de um curso superior em tecnologia; estudantes do quarto ano de um curso de Licenciatura em Matemática; alunos do Ensino Médio; professores dos anos iniciais e do Ensino Fundamental; docentes de Ciências e Matemática do Ensino Médio.

Durante a leitura integral dos artigos, tornou-se possível identificar a temática de pesquisa das produções referente à utilização de Objetos de Aprendizagem no ensino e na aprendizagem. Duas produções sobre aprendizagem de crianças com tecnologias, três trabalhos com o tema relação e interação entre tecnologias e professores de Ciências e Matemática, dois artigos com a temática tecnologia como proposta de inovação no ensino de conteúdos matemáticos e um abordando o ensino a distância e ambiente virtual de aprendizagem como reforço.

\subsection{Aprendizagem de crianças com tecnologias}

Com relação aos Objetos de Aprendizagem e recursos digitais das TIC que aparecem nos artigos como possibilidades de ensino e de aprendizagem, tem-se como aporte teórico, no artigo A3, Ferreira (2006) e Pagel (2012). Ferreira (2006) evidencia a importância de uma política pedagógica com atividades lúdicas no desenvolvimento infantil, além da valorização dos docentes que trabalhem com crianças. Pagel (2012) destaca a capacidade de aprender com os outros e a potencialidade da tecnologia em transmitir e construir o conhecimento.

No artigo A1, os autores com mais ênfase teórica são Almeida (2007), Campos et al. (2003) e Cunha Filho et al. (2000). Diante disso, Almeida (2007) ressalta a importância da cooperação no desenvolvimento dos indivíduos como alternativa eficaz para a aprendizagem. Campos et al. (2003) acrescentam que a aprendizagem cooperativa é uma estratégia pedagógica 
eficaz. Além disso, Cunha Filho et al. (2000) salientam a tecnologia como um instrumento que sustenta e facilita ainda mais o processo cooperativo de aprendizagem. Outro autor que aparece no artigo A1 é Vigotsky (1991). Para Vigotsky (1991), a aprendizagem é um procedimento pelo qual os sujeitos adquirem habilidades, valores e informações quando entram em contato com a realidade e outros indivíduos.

Nessa categoria, as pesquisas apresentaram resultados destacando os Objetos de Aprendizagem como um recurso tecnológico mediador nos processos de ensino e aprendizagem. Nesse sentido, é um instrumento que potencializa as formas de ensinar e aprender. Passou-se a verificar um progresso na maneira de como os alunos se expressam e uma melhor compreensão de conceitos e da simbologia matemática. Além disso, a inserção de tecnologias em atividades cooperativas entre crianças se mostrou eficaz como possibilidade de troca de informações entre estudantes. O uso de Objetos de Aprendizagem em trabalhos em grupos não se limitou em transmissão de informações, mas propiciou à criança ser o sujeito da própria aprendizagem e promoveu modificações positivas em relação às suas ações interpessoais, obtendo uma melhoria no seu desempenho escolar. Ou seja, as crianças confrontam suas ideias diante o posicionamento de seus colegas, testando e elaborando suas estratégias para alcançar o objetivo proposto.

\subsection{Relação e interação entre tecnologias e professores de Ciências e Matemática}

O artigo A4 traz um embasamento enfatizando que a internet é uma ferramenta muito importante e as TIC possuem uma capacidade de transformar o modo como os professores ensinam e os alunos aprendem, além de poder modificar a sala de aula em um ambiente mais cooperativo (HEIDE; STILBORNE, 2000). O A6 reforça que os professores possuem ferramentas disponíveis para a educação online (BORBA et al., 2008), porém, existe uma lacuna entre a formação dos professores e as habilidades e conhecimentos necessários que as novas tecnologias exigem (KENSKI, 2013). Em vista disso, no A8, Brasil (2013) aponta os instrumentos tecnológicos como aporte pedagógico às atividades escolares e a importância da formação continuada de professores com utilização de recursos digitais, como preparação na atuação com instrumentos tecnológicos.

Os artigos A4, A6 e A8 destacam as questões de pesquisa: como a tecnologia pode contribuir nas aulas? Os docentes possuem conhecimento e utilizam ferramentas tecnológicas? $\mathrm{E}$ de que forma as tecnologias digitais podem auxiliar no desenvolvimento profissional? Assim, as considerações ressaltaram que os usos de Objetos de Aprendizagem podem colaborar para o trabalho de conceitos novos e visualização de situações nunca presenciadas pelos estudantes. Sabendo das possíveis contribuições positivas das tecnologias, a maioria dos professores afirma que possuem uma relação "amistosa" com as TIC. Contudo, a proximidade com os recursos e 
Objetos de Aprendizagem se dá, no início, sem muita experiência, apesar de reconhecerem a potencialidade dessas tecnologias nos processos de ensino e de aprendizagem.

\subsection{Tecnologia como proposta de inovação no ensino de conteúdos matemáticos}

O artigo A7 apresenta referenciais que evidenciam os problemas com o ensino mecânico, aulas e explicações prontas (BRASIL, 1997). Bittar (2011) reforça que os professores devem ter contato e utilizar os recursos digitais em suas aulas. Assim, Bueno (2013) ressalta a necessidade de o docente investir na formação continuada, pois não basta só o recurso tecnológico para que ocorra aprendizagem. Borba e Penteado (2010) defendem que os conhecimentos são desenvolvidos e gerados por um coletivo formado por seres humanos com mídias digitais ou seres humanos com tecnologias. O A5 aponta a ideia de que o domínio dos sistemas digitais pelo professor influencia nas ações didáticas planejadas que, por conseguinte, o aluno constrói significados e desenvolve habilidades para aprender (BUSSI; MARIOTTI, 2008).

As pesquisas discutem diferentes abordagens de conceitos matemáticos e como torná-los mais interessantes utilizando Objetos de Aprendizagem em sala de aula. Assim, os resultados em evidência salientam as ferramentas digitais não só como possíveis maneiras de abordar conteúdos novos, mas como inovação nas propostas de ensino. As tecnologias podem transformar o ensino e a aprendizagem, porém depende de uma mudança na postura do professor para superar a aula expositiva e de reprodução. O docente pode aproveitar as vantagens dos recursos digitais para sugerir a realização de algo novo no ensino, além de fomentar o pensamento questionador de seus alunos em vez de trabalhar conteúdos "prontos".

\subsection{Ensino a Distância e ambiente virtual de aprendizagem como reforço}

O artigo A2 contempla embasamentos sobre educação em um ambiente virtual de aprendizagem. O EAD tende a ser um elemento padrão no âmbito educacional para atender as demandas e assumir funções na educação, visto que a criação de metodologias focadas no estudante e na sua autonomia é uma condição oportuna para a experiência EAD (BELLONI, 1999), além de ser outro meio de relação interpessoal (MOORE; KEARSLEY, 1996). A educação por meio de um ambiente virtual é um autoestudo, uma conversa indireta que estimula discussões sobre os conceitos em uma interação entre os estudantes (SEWART et al., 1991). Conforme a Teoria da Aprendizagem Cognitiva (AUSUBEL et al., 1980), o aluno desenvolve a sua capacidade de adquirir conhecimento de forma individualizada e independente (aprendizagem significativa).

Em relação às considerações do artigo $A 2$, destaca-se o EAD como um recurso tecnológico que possui um grande valor pedagógico. Essa ferramenta pode contribuir para que o aluno discuta suas dúvidas, levante problemas, interaja com outros alunos e questione os professores. Nesse sentido, engrandecendo e potencializando a sua aprendizagem. 


\section{Considerações finais}

O presente mapeamento teórico teve o intuito de identificar artigos acadêmicos inseridos no Banco de Periódicos da CAPES que contemplam os vocábulos Educação, Matemática, Tecnologia e o termo exato "Objetos de Aprendizagem" publicados entre os anos de 2010 e 2016. Nesse contexto, foi possível verificar as principais produções segundo o assunto escolhido como tema no campo da Educação Matemática.

Durante a análise dos artigos, foi possível verificar o tema de pesquisa, objetivos, embasamento teórico, metodologia, público alvo e principais resultados. Por meio do reconhecimento dos artigos escolhidos, notaram-se discussões sobre os OA, TIC, AVA e EAD como instrumentos que podem contribuir e potencializar modos de ensinar e de aprender. Essa concepção ficou facilmente perceptível nas oito pesquisas escolhidas em razão de abordarem a importância, a capacidade e a possibilidade que as tecnologias digitais têm de transformar os processos de ensino e de aprendizagem.

Acerca disso, evidenciou-se a emergência das seguintes categorias: aprendizagem de crianças com tecnologias; relação e interação entre tecnologias e professores de Ciências e Matemática; tecnologia como proposta de inovação no ensino de conteúdos matemáticos; ensino a distância e ambiente virtual de aprendizagem como reforço.

Diante dessas categorias, os OA foram evidenciados como uma ferramenta mediadora de conhecimento, inovadora de conteúdos, além de possuir ampla relevância pedagógica que pode contribuir positivamente na interação com o professor apesar de existir a necessidade da aproximação dos docentes a esses recursos. Entretanto, torna-se importante e necessária a realização de mais pesquisas que evidenciem o papel das TIC na transformação do ensino e da aprendizagem.

Finalizando, salienta-se que as observações e apontamentos expostos nesse mapeamento teórico poderiam ser diferentes se os artigos escolhidos para a análise fossem outros. Porém, isso não tira o mérito dessa pesquisa no campo da Educação Matemática em relação aos termos "Objetos de Aprendizagem", Educação, Matemática e Tecnologia.

\section{Referências}

ALMEIDA, A. R. S. A emoção na sala de aula. Campinas: Papirus, 2007.

AUSUBEL, D. P.; NOVAK, J. D.; HANESIAN, H. Psicologia Educacional. Rio de Janeiro: Interamericana, 1980.

BAIRRAL, M. A. Educação Matemática, Tecnologias Digitais e Educação a Distância: um olhar retrospectivo para os artigos do SIPEM. In: ROSA, Maurício; BAIRRAL, Marcelo Almeida; AMARAL, Rubia Barcelos. Educação Matemática, Tecnologias Digitais e Educação a Distância: pesquisas contemporâneas. Natal: Livraria da Física, 2015. p. 97-130. 
BUSSI, M. G. B.; MARIOTTI, M. A. Semiotic mediation in the mathematics classroom: artifacts and signs after a Vygotskian perspective. In: ENGLISH, L. et al. (Eds.). Handbook of International Research in Mathematics Education, second revised edition, Lawrence Erlbaum: Mahwah, NJ. 2008. p. 746-783.

BELLONI, M. L. Educação a distância. Campinas, SP: Autores Associados, 1999.

BICUDO, M. A. V. et al. Educação matemática. São Paulo: Moraes, 2005.

BITTAR, M. A abordagem instrumental para o estudo da integração da tecnologia na prática pedagógica do professor de matemática. Educar em Revista, n. 1, p. 157-171, 2011.

BIEMBENGUT, M. S. Mapeamento na pesquisa educacional. Rio de Janeiro: Ciência Moderna, 2008.

BORBA, M. C.; MALHEIROS, A. P. dos S.; ZULATTO, R. B. A. Educação a distância online. 2. ed. Belo Horizonte: Autêntica, 2008.

BORBA, M. C.; PENTEADO, M. G. Informática e Educação Matemática. 4. ed. Belo Horizonte: Autêntica, 2010.

BOUCINHA, R. M.; GRASEL, P.; TAROUCO, L. M. R. Desenvolvimento de objetos de aprendizagem multimodais para tablets. In: DE LIMA, José Valdeni et al. Objetos de Aprendizagem Multimodais: Projetos e Aplicações. Editorial UOC, 2014. p. 165-178.

BRASIL. Ministério da Educação. Parâmetros Curriculares Nacionais: matemática. Brasília: MEC/SEF, 1997.

BRASIL. Ministério da Educação. Secretaria de Educação Básica. Secretaria de Educação Continuada, Alfabetização, Diversidade e Inclusão. Secretaria de Educação Profissional e Tecnológica. Conselho Nacional da Educação. Câmara Nacional de Educação Básica. Diretrizes Curriculares Nacionais Gerais da Educação Básica. Brasília, 2013.

BUENO, N. L. Tecnologia educacional e reificação: uma abordagem crítica a partir de Marxs e Luckás. 2013. 503f. Tese (Doutorado em Educação) - Universidade Federal do Paraná, Curitiba, 2013.

CAMPOS, F.; SANTORO, F. M.; BORGES, M. R. S. Cooperação e aprendizagem online. Rio de Janeiro: Co\&a, 2003.

CUNHA FILHO, P.; NEVES, A.; PINTO, R. O projeto Virtus e a Construção de Ambientes Virtuais de Estudo Cooperativos. In: MAIA, C. Educação a distância no Brasil na era da Internet. São Paulo: Anhembi Morumbi, 2000. p. 53-72.

FABRE, M. C. J. M.; TAMUSIUNAS, F.; TAROUCO, L. M. R. Reusabilidade de objetos educacionais. RENOTE, v. 1, n. 1, 2003.

FERREIRA, C. A. de M. A relação entre o brincar, a consciência e o desenvolvimento, sob uma ótica vygotskiana. Revista Eletrônica Informação e Cognição, v. 5, n. 1, p. 137-180, 2006.

FILHO, A. B. do C.; LIMA, J. V. de. Princípios de projeto. In: DE LIMA, José Valdeni et al. Objetos de Aprendizagem Multimodais: Projetos e Aplicações. Editorial UOC, 2014.

HEIDE, A.; STILBORNE, L. Guia do Professor para a Internet - completo e fácil. 2. ed. Porto Alegre: Artes Médicas Sul, 2000. 
KENSKI, V. M. Tecnologias e tempo docente. Campinas, SP: Papirus, 2013.

KIRNER, C.; SISCOUTTO, R. Realidade virtual e aumentada: conceitos, projeto e aplicações. In: Livro do IX Symposiumon Virtual andAugmented Reality. Petrópolis, Editora SBC. 2007.

MOORE, M.; KEARSLEY, G. Distance Education: a systems view. Belmont: Wadsworth, 1996.

OLIVEIRA, C. TIC's na Educação: A utilização das Tecnologias da Informação e Comunicação na aprendizagem do aluno. Pedagogia em Ação, v. 7, n. 1, 2015.

PAGEL, M. Adapted to culture. Nature, London, 482, 7385, 297-299, Feb. 2012.

PAULIN, J. F. V. Educação Matemática, Tecnologias Digitais e Educação a Distância: um olhar retrospectivo para os artigos do SIPEM. In: ROSA, Maurício. BAIRRAL, Marcelo. Almeida. AMARAL, Rubia. Barcelos. Educação Matemática, Tecnologias Digitais e Educação a Distância: pesquisas contemporâneas. Natal: Livraria da Física, 2015. p. 17-56.

PICCOLI, L. A. P. A Construção de Conceitos em Matemática: uma proposta usando tecnologia de informação. 2006. 109f. Dissertação (Mestrado em Educação em Ciências e Matemática) PUCRS, Porto Alegre, 2006.

SEWART, D.; KEEGAN, D.; HOLMBERG, B. Distance Education International Perspectives. London: Routllege, 1991.

SINGO, F. Objetos de aprendizagem multimodais. In: DE LIMA, José Valdeni et al. Objetos de Aprendizagem Multimodais: Projetos e Aplicações. Editorial UOC, 2014. p. 17-34.

TAROUCO, L. M. R.; FABRE, M. C. J. M.; KONRATH, M. L. P; GRANDO, A, R. Objetos de Aprendizagem para M-learning. In: Florianópolis: SUCESU-Congresso Nacional de Tecnologia da Informação e Comunicação. 2004.

VYGOTSKY, L. S. Pensamento e Linguagem. 4. ed. São Paulo: Martins Fontes, 1991. 